\title{
基础有机化学教学中的课程思政教育设计 有机化学发展简史中的课程思政元素
}

\author{
武全香 ${ }^{*}$, 惠新平 \\ 兰州大学化学化工学院, 兰州 730000
}

\begin{abstract}
摘要: 把思想政治工作贯穿于整个教育教学全过程是新时代高等教育的重要任务, 有机化学课程教学与素质教育、 思想政治教育相结合, 培养具有高度社会责任感的化学专业人才是高校化学教育的目标。从有机化学发展史的三个 阶段和我国有机化学发展等方面, 介绍了兰州大学在基础有机化学课程教学中, 通过中华传统文化、名人励志故事 等内容, 梳理了有机化学发展史中蕴含的思想政治教育元素, 引导学生在学习基础知识、了解历史的同时, 培养正 确的世界观、人生观和价值观。
\end{abstract}

关键词: 有机化学; 课程思政; 发展简史

中图分类号：G64; O6

\section{Course Ideology and Politics in Basic Organic Chemistry Teaching: A Concise History of Organic Chemistry}

\author{
Quanxiang Wu *, Xinping Hui \\ College of Chemistry and Chemical Engineering, Lanzhou University, Lanzhou 730000, China.
}

\begin{abstract}
It is an important task for higher education in the new era to put course ideology and politics through the whole teaching process. The goal of chemical education, combining course teaching with quality education and ideological and political education, in colleges and universities, is to cultivate chemical professionals with a high sense of social responsibility. This paper introduces the ideological and political education elements contained in the history of organic chemistry in China and the world. The traditional Chinese culture and inspirational stories of celebrities are introduced. The purpose is for students to learn basic knowledge and understand history. At the same time, the aim is to cultivate a correct world outlook on life and values.
\end{abstract}

Key Words: Organic chemistry; Course ideology and politics; Concise history

新时代背景下大学生思想政治教育已成为高等教育的重要任务, 教师在传授专业知识和培养专 业素养的同时，必须开展思想政治教育，把立德树人作为中心环节，把思想政治工作贯穿于整个教 育教学全过程, 实现全程育人、全方位育人 ${ }^{[1]}$ 。有机化学是主要研究有机化合物的组成、结构、性 质、制备(合成)方法、功能和应用的科学, 是化学学科的重要分支之一。有机化学教学不仅传授专业 基础知识、基本理论和基本技能, 引导学生了解前沿新知识、新理论和新方法, 培养学生具有较强 实践能力、分析和解决问题能力、创新能力和批判性思维能力; 同时通过课程教学与素质教育、思

收稿: 2020-11-03; 录用: 2020-11-23; 网络发表：2020-12-11

“通讯作者, Email: wuqx@1zu.edu.cn

基金资助: 兰州大学有机化学课程思政示范课程和教学改革示范课程建设项目 
想政治教育相结合, 融入鲜明的时代特征和新时代的社会需求, 培养具有高度社会责任感的化学专 业人才，为民族复兴提供人才支撑 ${ }^{[2]}$ 。

有机化学与人类社会发展息息相关, 经过几百年的发展才有了今天的辉煌。著名的化学教育家 傅鹰(1902-1979)先生说过 “化学可以给人以知识, 化学史可以给人以智慧”。有机化学发展史提供了 有机化学发展的来龙去脉, 是科学发现、技术发明的历史。学习有机化学发展史, 了解雄伟悲壮的 重大事件, 可以使我们树立正确的自然观和科学观。在基础有机化学教学中, 我们将课程思政教育 融进教学大纲、写入教案、引入课堂, 深入挖掘课程思政教育案例, 设计课程思政教育环节, 找准 思政教育切入点, 实施了多样化、嵌入式思政教育。下面将我们在有机化学发展简史中的课程思政 元素做以简单介绍。

\section{1 早期萌芽时期}

\section{1 中国使用有机物的悠久历史}

最初的有机物是由动植物有机体中得到的物质。中华文明是辉煌灿烂的, 在很长的时间里, 它 一直走在世界前列, 使用有机物造福人类有着光辉的篇章。《神农本草经》是世界上最早的药典, 记 载了 365 种药物的疗效, 多数为植物药。中国有六千年的酒文化历史, 虽然关于酒的起源众说纷纭, 但都是由粮食、花、果等发酵而来。我国是世界上谷物酿醋最早的国家，南北朝时的《齐民要术》 系统总结了我国劳动人民从上古到北魏时期的制醋经验和成就, 共收载了 22 种制醋方法。西汉时期 的造纸术是中国古代四大发明之一, 纸一般由经过制浆处理的植物纤维水悬浮液, 经脱水、压缩、 烘干等工序而成。据《史记·货殖列传》记载, 我国早在周朝时已经开始大量生产秨(蓝色)、茜(红色)、 姜(黄色)、韭(绿色)等植物染料 ${ }^{[3]}$ 。

这些植物药、酒、醋、染料等都是有机混合物, 是中国劳动人民长期经验的积累, 是集体劳动 和智慧的结晶。从中华民族的悠久历史和传统文化, 延伸到有机物的使用, 让学生了解中华民族对 人类文明的巨大贡献、认识传统文化所蕴含的化学知识、体会化学在人类衣食住行中的重要性, 从 而培养学生关注生活中的化学, 培养文化认同感, 增强民族自信力。

\section{2 生命力学说}

17-18世纪, 人类从动植物中获得了许多有机化合物(见图1), 如1769年舍勒(C. W. Scheele, 17421786, 瑞典化学家)从酿酒副产品酒石中分离出酒石酸(1); 1773年罗埃尔(H. M. Rouelle, 1718-1779, 法国化学家)用乙醇萃取哺乳动物尿液中的物质, 蒸发结晶得到一种白色物质, 其中的主要成分是尿 素(2); 1805年塞尔杜纳(F. Sertürner, 1783-1841, 德国化学家)从鸦片中分离出了第一个生物碱吗啡 (3), 并在狗身上进行了麻醉试验。
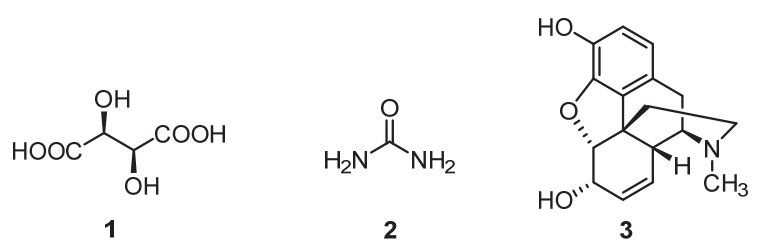

图1 酒石酸、尿素和吗啡的结构

19世纪初, 碳化合物的化学比金属和氮、硫、磷等的化学远为落后。1806年有机化学之父一 贝采里乌斯(J. J. Berzelius, 1779-1848, 瑞典化学家)首次提出了 “有机化学”, 当时是作为 “无机化 学” 的对立物而命名。由于受当时的条件限制, 有机化学研究的对象只能是天然动植物有机体, 因 而被分为植物化学和动物化学。许多化学家因此认为, 在生物体内存在特殊的、神秘的 “生命力” 才能产生有机化合物, 这些物质不能用无机物合成 ${ }^{[3-7]}$ 。

有机化学起源于这一阶段天然有机化学的发展, 由于认识的局限性和对权威的迷信, “生命力” 
学说曾一度牢固地统治着有机化学界, 严重阻碍了有机化学其他领域的发展。通过介绍生命力学说, 引领学生认识到化学的重大发现都有其历史的必然性, 都是在人民群众所提供的物质、技术、生活 条件的基础上取得的, 从事物质生产的广大劳动群众是推动科学技术进步的真正动力。

\section{3 有机化学是碳的化学}

1828年维勒(F. Wohler, 1800-1882, 德国化学家)无意中用加热的方法使无机化合物异氧酸铵(4) 转化为尿素(图2), 这一重要发现并未马上得到权威化学家的承认。直到 1845 年柯尔伯(H. Kolbe, 1818-1884, 德国化学家)利用木炭、硫磺、氯气及水为原料合成了醋酸; 1854年柏赛罗 (M. Berthelot, 1827-1907, 法国化学家)利用石蜡合成了油脂, 生命力学说才被彻底抛弃。从此开创了有机合成化 学的新时代, 人类不仅可以合成天然有机化合物, 而且可以制备由生物体无法得到的、用途更广、 结构新颖的有机化合物。有机合成化学的迅速发展使人们认识到, 有机物与无机物之间并没有明确 的界限, 但在组成和性质上确有不同之处。1848年葛美林(L. Gmelin, 1788-1853, 德国化学家)重新 定义了有机化学即为研究碳化合物的化学 ${ }^{[3-7]}$, 这标志着有机化学作为一门学科的诞生, “有机化学” 这一名词沿用至今。

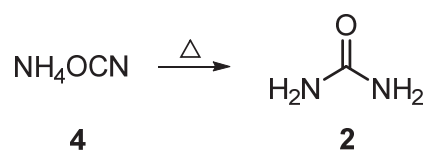

图2 由异㝘酸铵合成尿素

化学是一门实验学科, 化学实验是科学家认识物质、改变物质的重要手段。讲解有机化学定义 的发展, 从生命力学说到维勒合成有机物, 让学生认识到有机化学发展史也是人类进步史, 只有提 升创新意识, 才能适应新时代的发展需要。只有敢于打破习惯思维和主观偏见, 以更宽阔的眼界、 思路、胸襟，敢于自我否定，才能创造性地提升学习与工作的水平和质量。

\section{2 经典有机化学时期}

\section{1 凯库勒和库珀关于碳的两个重要基本规则}

19世纪中期, 化学结构不可知论非常流行, 直到1858年凯库勒(F. A. Kekule, 1829-1896, 德国 化学家)和库珀(A. S. Couper, 1831-1892, 英国化学家)分别独立提出 “碳原子是四价的, 并能相互连

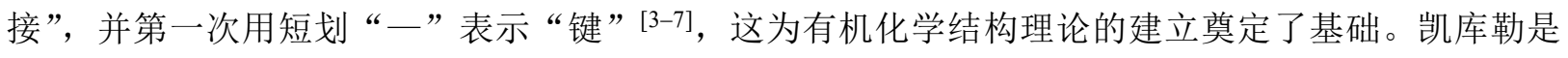
一位极富想象力的学者, 在提出了多种有关苯的开链式结构而又因其与实验结果不符被一一否定之 后, 据他自己说, 1865年的一天夜晚, 在书房中打瞌睡的他眼前出现了旋转的碳原子长链像蛇一样 盘绕卷曲, 忽见一蛇抓住了自己的尾巴, 并旋转不停。他像触电般地猛醒过来, 整理苯的结构假说, 提出闭合链形式是解决苯分子结构的关键, 他以 5 式表示苯的结构。1866年他又提出苯分子是由六个 碳原子以单、双键交替结合而成的环状结构6, 后简化为7式, 也就是所说的凯库勒式(图3)。<smiles>c1ccccc1</smiles>

5<smiles>c1ccccc1</smiles>

6<smiles>c1ccccc1</smiles>

7

图3 凯库勒表示苯的环状结构式

凯库勒从梦中得到启发, 成功提出了苯的重要结构学说, 其实并非偶然。这与他善于独立思考, 平时冥思苦想, 才会梦其所思; 更重要的是, 他善于捕捉直觉现象, 加之以事实为依据, 以科学态 
度进行多方面的分析和探讨, 这一切为他取得成功奠定了基础。通过介绍科学家的故事, 引领学生 学习他敢于实践、大胆创新的科学精神。任何一种科学发现都有其必然性, 多思考、勤实践, 才能 在科学的道路上勇攀高峰。

\section{2 范特霍夫和勒比尔的碳原子四面体构型}

凯库勒的经典结构理论仅仅提出了分子中原子的原子价、数目、种类和关系等, 1861年布特列 洛夫(A. M. Butlerov，1828-1886, 俄国化学家)首次提出了化学结构的概念, 在很长一段时间内, 人 们运用物质的化学性质去推测化学结构。1874年范特霍夫(J. H. van't Hoff, 1852-1911, 荷兰化学家) 和勒比尔(J. A. LeBel, 1847-1930, 法国化学工艺师)提出了碳原子的四面体构型学说, 之后又提出 了同分异构体的概念, 建立了有机立体化学的结构, 并阐明了旋光异构现象 ${ }^{[3-7]}$ 。维斯利采纽斯 (J. Wislicenus, 1835-1902, 德国有机化学家)在此基础上研究了旋光乳酸 $\left(\mathbf{8 a}\right.$ 和 $\mathbf{8 b}$ ) 的结构 ${ }^{[4]}$, 为该异 构现象提供了实例(图4)。

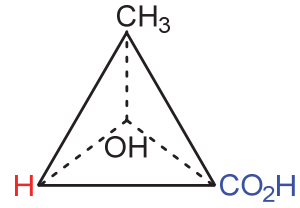

$8 a$

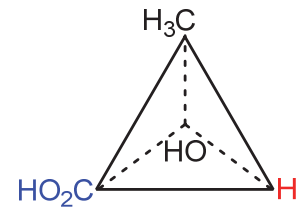

$8 b$

图4 乳酸的立体异构体

过去的有机结构理论认为有机分子中的原子处在一个平面内, 这与很多现象相矛盾。范特霍夫 (波恩大学, 凯库勒的学生)纠正了该错误, 首先提出了碳的四面体结构学说, 但这一新的理论却遭到 了一些权威人士的反对。当时德国化学家柯尔伯质疑他的理论时, 范特霍夫恭恭敬敬地等候, 平心 静气地介绍自己的实验结果, 陈述自己的观点。范特霍夫实事求是、谦虚谨慎的态度使柯尔伯心悦 诚服地接受了他的理论, 并邀请范特霍夫去普鲁士科学院工作。1901年, 范特霍夫成为第一位诺贝 尔化学奖获得者。有趣的是, 范特霍夫创立的碳四面体结构学说并不是获奖原因, 而是他的另外两 篇著名论文《化学动力学研究》和《气体体系或稀溶液中的化学平衡》。

范特霍夫这种勤于观察、善于思考以及重视科学实验的精神值得我们学习。他敢想敢做的创新 精神, 对我们是一种莫大的鞭策; 他实事求是的态度, 豁达友善的为人, 摆事实、讲道理更容易让 人心悦诚服。

\section{3 第一个自由基的发现}

1900年, 冈伯格(M. Gomberg, 1866-1947, 美国化学家)发现了稳定的三苯甲基自由基, 开启了 人类自由基研究先河。他用纯银或锌在苯等惰性溶剂中处理三苯氯甲烷(9)得到了白色固体(图5) ${ }^{[8-10]}$ 。 冈伯格认为该白色固体是三苯氯甲烷的偶联产物六苯乙烷(11)。但随后发现该产物比他想象中的六 苯乙烷活泼的多, “六苯乙烷” 在溶液中部分分解为自由基 $\mathbf{1 0}$, 如它的苯和醚溶液呈黄色, 若迅速振 荡则黄色消失, 过几秒钟后又出现黄色, 继续振荡黄色又消失。这是最早存在自由基的报道, 尽管 当时关于自由基的说法还不为化学家所接受。1904年有人提出 “六苯乙烷” 的结构实际上为醌式结 构 12, 但这个说法不为当时的大多数化学家所接受, 直到1968年人们使用核磁共振谱和紫外光谱方 法研究上述平衡体系, 发现所谓 “六苯乙烷” 确为醌式结构 ${ }^{[11]}$ 。

1954年, 中国自由基化学的奠基人、中国科学院院士、兰州大学刘有成教授(1920-2016, 师从 美国化学家卡拉施教授(M. S. Kharasch，1895-1957), 自由基化学奠基人之一, 提出了反马氏加成规 则)为了报效祖国, 放弃国外优越的科研生活条件, 服从分配, 到兰州大学工作。他克服困难, 扎根 祖国西北, 满腔热情投入教学科研, 为祖国培养了一大批优秀的有机化学人才。在他和其他老师的 共同努力下, 1983年兰州大学有机化学学科被评为国家重点学科(当时的有机化学重点学科只有北京 

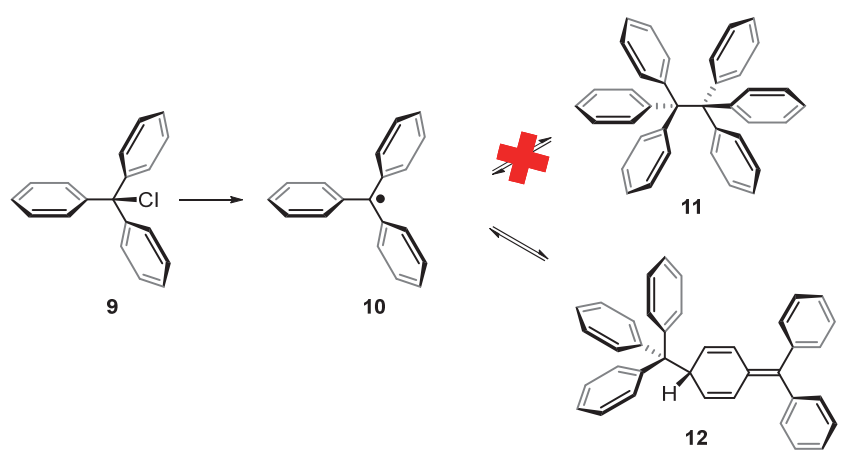

图5 三苯甲基自由基的形成及结构

大学、南开大学和兰州大学), 1985年国家计委在兰州大学设立了 “应用有机化学国家重点实验室” (2013年改名为 “功能有机分子化学国家重点实验室” ), 为我国首批国家重点实验室。

刘有成先生的事迹充分体现了以爱国主义为核心的民族精神, 作为新时代的青年, 我们不仅要 发扬老一辈化学家勤于思考、不断探索的科学精神, 更要具有深厚的家国情怀, 努力为国家化学事 业的发展与进步贡献自己的力量。

\section{3 现代有机化学时期}

\section{1 共价键电子理论}

19世纪初有机化合物在结构测定、反应和分类等方面取得了很大进展, 但价键只是化学家从实 践经验得出的一种概念, 价键的本质问题尚未解决。直到价键电子理论的提出, 才解开了有机化合 物结构的谜团。

在物理学阐明原子结构的基础上，1916年路易斯(G. N. Lewis，1875-1946, 美国化学家)提出了 经典的共价键理论(8电子规则), 两个原子共用外层电子形成共价键。通过电子的转移或共用, 使相 互作用的原子的外层电子都获得惰性气体的电子构型 ${ }^{[3-7]}$ 。Lewis规则可以初步解释大多数主族元素 的成键情况, 也可分析分子的几何结构。但Lewis结构不能解释共价键的本质和特性, 特别是不能解 释 “共用两个电子就能使原子结合成分子”。但八隅体规则也有例外, 特别是从第三周期开始的元素 以及硼元素、部分氮的氧化物等。

介绍在物理学发现的基础上, 路易斯提出共价键理论, 引导学生及时关注相关专业前沿研究动 态和进展, 关注科学中的每一个新发现, 关注社会进步, 才能紧跟时代潮流、与时俱进, 不断开拓 创新。

\section{2 杂化轨道理论}

1922-1933年, 物质结构研究发生了巨大变化, 量子力学在化学中得到了应用, 鲍林(L. Pauling, 1901-1994, 美国化学家)利用这一理论研究了原子和分子的电子结构以及化学键的本质。1928年, 鲍林根据碳原子与氢原子的结合方式和碳原子中电子分层排布的事实 ${ }^{[5]}$, 通过科学的想象和逻辑推 理, 创造性地提出了 “杂化轨道” 理论, 比较圆满地解决了碳在有机化合物中是怎样以 4 价结合的问 题(图6)。

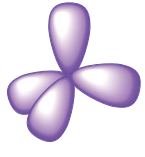

$13 a$

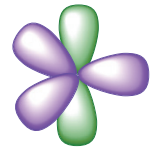

$13 b$

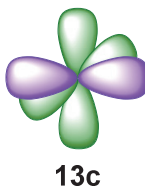

$13 c$

图6 碳原子 $s p^{3}(13 a) 、 s p^{2}(13 b) 、 s p(13 c)$ 杂化轨道 
鲍林这一创造性理论很好地解释了有机化合物中化学键的本质问题, 为有机结构和理论化学的 发展做出了重要贡献。但鲍林的 “杂化轨道” 理论也遇到了新的挑战。例如后来人们合成了结构和 性质较稳定的环丙烷、环丁烷、立方烷等, 它们的键角很难用 “杂化轨道” 理论进行解释。现今人 们对化学键的认识还很不够, 还需要开展新的研究, 科学的发展往往是旧的矛盾解决了, 新的矛盾 又产生了。

\section{3 现代波谱技术}

波谱技术出现之前, 用化学分析法确定物质的相对分子质量、分子式、结构片段及相互关系是 非常困难的。如 1805 年人类已经从鸦片中提取了吗啡, 经历了 120 年, 直到 1925 年才确定了其结构。

波谱法主要包括红外光谱、紫外光谱、核磁共振谱和质谱, 此外还包括拉曼光谱、荧光光谱、 旋光光谱、圆二色谱、顺磁共振谱等。从19世纪中期至今, 波谱分析经历了漫长的发展过程。19世 纪末人们开始使用红外光谱和紫外光谱测定化合物的结构, 1912年出现了第一台质谱仪, 并用其发 现了 $\mathrm{Ne}$ 的两种同位素。1945年发现了核磁共振现象, 此后核磁共振技术在化合物的结构解析中得到 了广泛应用。随着计算机技术的飞速发展, 波谱分析技术不断完善和创新, 在方法、原理、仪器设 备和应用上得到了突飞猛进的发展。20世纪60年代以后, 现代波谱技术的发展大大简化了结构研究 ${ }^{[12]}$ 。

化学是从事化学实验的广大化学工作者和科研人员创造出来的, 分析仪器的更新和换代已使有 机化学成为一项较为系统的研究工作。化学与其他科学技术一样, 本身也是一种生产力。它可以直 接参与到生产活动中去, 促进生产力的飞跃, 推动社会的进步。通过介绍波谱技术发展, 让学生认 识到波谱技术的发展推动了化学的日新月异，化学推动了波谱技术的继续进步，两者相辅相成。

\section{4 我国有机化学发展}

中国的有机化学起步较晚, 与欧美国家的科研历史相比, 差了一个半世纪, 而中间又受到各种 因素的干扰, 发展缓慢, 但发展趋势与欧美国家一致。20世纪20年代是中国有机化学研究的起点, 之后有机化学的各个研究方向逐渐发展起来(表1) ${ }^{[13]}$ 。

表1 我国有机化学的研究方向及开始时间

\begin{tabular}{cc}
\hline 开始时间 $(20$ 世纪) & 研究方向 \\
\hline 20 年代 & 天然有机化学 \\
30 年代后期 & 有机微量分析 \\
30 年代末期 & 有机合成化学 \\
30 年代 & 金属有机化学 \\
40 年代 & 紫外光谱分析 \\
50 年代 & 物理有机化学 \\
50 年代后期 & 元素有机化学、红外光谱分析 \\
60 年代中期 & 核磁共振波谱分析 \\
70 年代初期 & 质谱分析 \\
\hline
\end{tabular}

20 世纪 20 年代我国专门从事有机化学研究的科学家不过20余人, 庄长恭(1894-1962)、赵承嘏 (1885-1966)、黄鸣龙(1898-1979)、纪育沣(1899-1982)、曾昭抢(1899-1967)、杨石先(1897-1985)等是 中国第一代有机化学家, 他们对我国有机化学的发展做出了卓越贡献。庄长恭教授是我国有机化学 的先驱, 在有机合成方面做出了卓越贡献, 在国际有机化学界享有盛誉。曾昭抢教授是我国化学界 最早提倡高校应搞科研的人, 他合成的对亚硝基酚载入了《有机化学辞典》。赵承嘏教授对植物化 学, 特别是生物碱的分离结晶有独到之处, 他从 30 多种中药中发现了许多生物碱。黄鸣龙教授发明 
的 “黄鸣龙还原法” 在有机合成和结构测定中得到了广泛应用, 并写入了有机化学教材。

新中国成立后, 我国的有机化学取得了很大发展, 最引人注目的是 1965 年牛胰岛素的全合成, 这是世界上第一个人工合成的蛋白质。1978年以后, 我国的有机化学发展迅速, 1981年实现了核糖 核酸的全合成。目前, 我国已拥有相当规模的科研队伍、一批国际知名的科学家和重要的科研成果。

与此同时, 兰州大学的有机化学研究也取得了长足进展, 20世纪50-60年代, 刘有成、朱子清 (1900-1989)、黄文鬼(1928-1982)、陈耀祖(1927-2000)等专家学者陆续来到兰州大学, 朱子清先生的 贝母植物碱研究曾居国际领先地位; 黄文魁教授的贝母植物碱的研究、碘杂环化合物的研究、三尖 杉酯类生物碱的合成研究和固氮酶活性中心化学模拟物的合成研究居同期国际同类研究的领先地 位; 陈耀祖院士主要进行质谱、质谱与色谱联用技术、天然药物结构分析、有机化合物微量系统鉴 定研究。他们为兰州大学有机化学学科的发展奠定了基础, 做出了突出贡献。

讲解中国有机化学的发展历史, 介绍兰州大学有机化学发展中做出卓越贡献的科学家, 使学生 认识到中国有机化学工作者从起步到辉煌所走过的艰辛漫长发展之路。了解兰州大学有机化学的发 展历史, 特别是改革开放以后, 有机化学学科迎来了前所未有的发展机遇, 使学生为母校取得的辉 煌成绩感到骄傲, 同时提高了他们的专业认同感和自豪感。

\section{5 结语}

美国化学家西博格说: 化学是人类进步的关键。两个多世纪以来, 有机化学研究不断深入, 科 学家们逐步揭示了有机分子中原子键合的本质以及有机化合物相互转化的规律, 设计、合成了具有 特定功能的有机分子, 为生命科学、医学、药学、农学、材料、能源、环境等学科奠定了基础。有 机化学在推动科技进步、社会进步, 提高人们生活质量, 改善环境的努力中, 已经并将继续发挥高 度开创性和解决重大问题的能力。

学习有机化学发展简史, 通过嵌入课程思政教育元素, 让学生认识到凡是有所作为的化学家, 都是百折不回、不辞辛苦、勇于献身科学的人。通过学习化学史, 了解为化学发展做出伟大贡献的 化学家的事迹, 尊重他们的劳动、智慧和成果, 学习他们分析问题与解决问题的思想和方法, 学习 他们在困难面前百折不挠的顽强毅力, 学习他们在科学道路上坦率无私、团结友爱、互帮互助的精 神。用科学家的事迹培养学生的科学精神, 启迪学生探究学习的灵感。

\section{参考文 献}

[1] 习近平: 把思想政治工作贯穿教育教学全过程. [2020-08-13]. http://www.xinhuanet.com//politics/2016-12/08/c_1120082577.htm

[2] 惠新平, 武全香. 大学化学, 2020,35 (7), 77.

[3] 张德生. 化学史简明教程. 合肥: 中国科学技术大学出版社, 2009: 85-103.

[4] Partington, J. R. 化学简史. 胡作玄, 译. 北京: 中国人民出版社, 2010: 174-256.

[5] Hirota, N. A History of Modern Chemistry; Kyoto University Press: Yoshida, Japan, 2016; pp. 224-580.

[6] 邢其毅, 裴伟伟, 徐瑞秋, 裴坚. 基础有机化学(上册). 第4版. 北京: 北京大学出版社, 2016: 1-26.

[7] 高坤, 李瀛, 王清廉, 张炜. 有机化学(上册). 北京: 科学出版社, 2007: 1-19.

[8] Gomberg, M. J. Am. Chem. Soc. 1900, 22 (11), 757.

[9] Gomberg, M. J. Am. Chem. Soc. 1901, 23 (7), 496.

[10] Gomberg, M. J. Am. Chem. Soc. 1902, 24 (7), 597.

[11] Lankamp, H.; Nauta, W. T.; MacLean, C. Tetrahedron Lett. 1968, 9 (2), 249.

[12] 常建华, 董绮功. 波谱原理及解析. 第3版. 北京: 科学出版社, 2012, 1-2.

[13] 郭保章. 20世纪化学史. 南昌: 江西教育出版社, 1998: 346-441. 